\title{
Hemichorea-hemiballismus as a rare presentation of non-ketotic hyperglycemia: a case report
}

\author{
Vijay Kumar*, Kotha Vamshi Krishna Patel, Rishav Raj, Divendu Bhushan, Aniketh V. Hegde
}

Department of General Medicine, All India Institute of Medical Sciences (AIIMS), Patna, Bihar, India

Received: 29 November 2021

Accepted: 29 December 2021

*Correspondence:

Dr. Vijay Kumar,

Email: vk1994@gmail.com

Copyright: (C) the author(s), publisher and licensee Medip Academy. This is an open-access article distributed under the terms of the Creative Commons Attribution Non-Commercial License, which permits unrestricted non-commercial use, distribution, and reproduction in any medium, provided the original work is properly cited.

\begin{abstract}
Hemichorea-hemiballismus is a hyperkinetic movement disorder on one side of the body resulting from involvement of the contralateral basal ganglia and striatum mainly. A 50-year-old female presented with a 5-days history of choreiform movements affecting the left upper and lower limb along with hemiface. Her plasma glucose was $576 \mathrm{mgl} / \mathrm{dl}$ and her urinary ketones were negative suggesting non-ketotic hyperglycemia. Computed tomography revealed hyperdensity in right caudate and putamen consistent with diabetic striatopathy. Non-ketotic hyperglycemia is a rare cause of hemichorea-hemiballismus syndrome. Hemichorea-hemiballismus can be an unusual and rare presentation of diabetic elderly population especially females. Early diagnosis and treatment of hyperglycemia yields an excellent prognosis.
\end{abstract}

Keywords: Hemichorea-hemiballismus, Nonketotic hyperglycemia, Diabetic striatopathy

\section{INTRODUCTION}

Hemichorea-hemiballismus is a hyperkinetic movement disorder that is characterized by unilateral continuous or intermittent, non-rhythmic, non-repetitive, abrupt jerky and involuntary movements involving both proximal and distal movements due to lesions of contralateral striatum. ${ }^{1}$ The basal ganglia controls body movements via Thalamocortical tracts and any pathology affecting this structure, is usually associated with a group of different movement disorders including Chorea (relatively lower amplitude affecting both proximal and distal movements) and Ballismus (relatively higher amplitude affecting proximal movements). ${ }^{2}$ This condition is more common among patients with diabetes, elderly population and in females. ${ }^{1}$

Although there are various structural lesions that are associated with Hemichorea-hemiballismus, the most common among them being a cerebrovascular disease either an Infarct or a haemorrhage of basal ganglia and thalamus. Next most common cause is non-ketotic hyperglycaemia. ${ }^{3}$ Other causes of chorea include chorea associated with infections, rheumatic chorea, thyrotoxicosis, systemic lupus erythematosus, systemic vasculitis, autoimmune etiologies, hypoxic encephalopathy and drug induced or substance abuse. ${ }^{2}$ The unique feature of non-ketotic hemichorea is its tendency for unilateral involvement while Chorea due to other metabolic causes has a tendency to involve bilaterally. ${ }^{4}$ Hemichorea-hemiballismus as a presentation of non-ketotic hyperglycemia in patients with diabetes, is rare and we are presenting hemichorea-hemiballismus as a case report in an elderly female with recent onset diabetes mellitus.

\section{CASE REPORT}

A fifty-year-old female was admitted with a history of abnormal involuntary movements for last five days involving left upper and lower extremities associated with 
left hemifacial-dyskinesia and intermittent involuntary movement of tongue. Two months ago, she was diagnosed as a case of diabetes mellitus but was not taking any medications for the same, since the time of diagnosis.

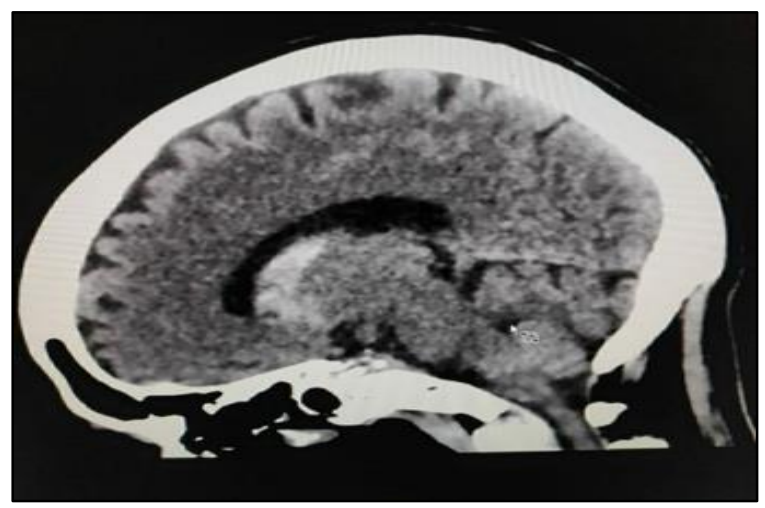

Figure 1: CT brain- revealed hyper-density of right caudate and right putamen.

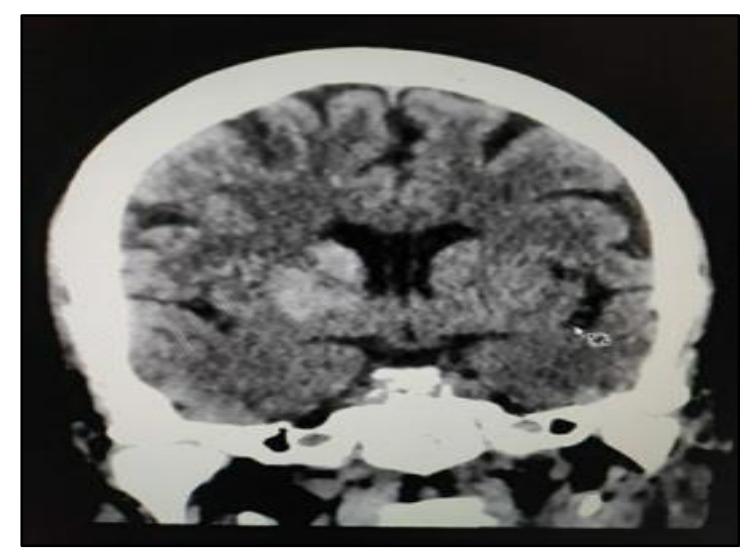

Figure 2: CT brain- revealed hyper-density of right caudate and right putamen.

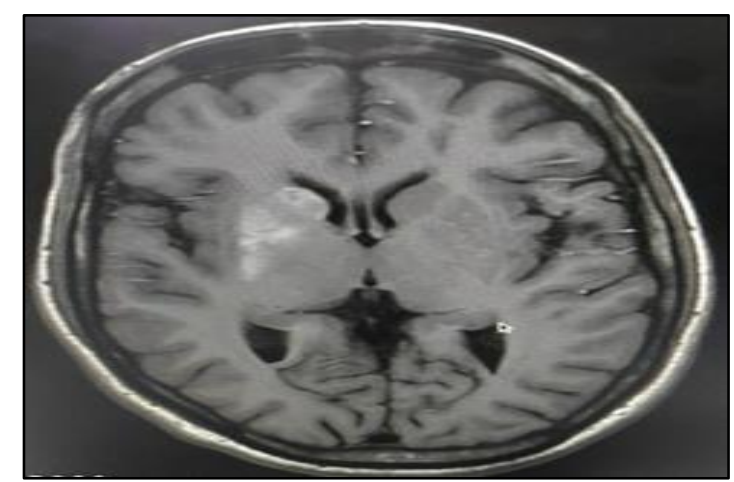

Figure 3: MRI brain- axial sections showing hyperintensity and hypo-intensity of the right caudate and right- putamen on $\mathrm{T} 1 \mathrm{~W}$.

These movements were acute in onset, jerky, hyperkinetic, random and purposeless. There is no past history of cerebrovascular disease, altered sensorium, diplopia, limb weakness, deviation of face to one side, slurring of speech, or slow writhing movements or tremors.

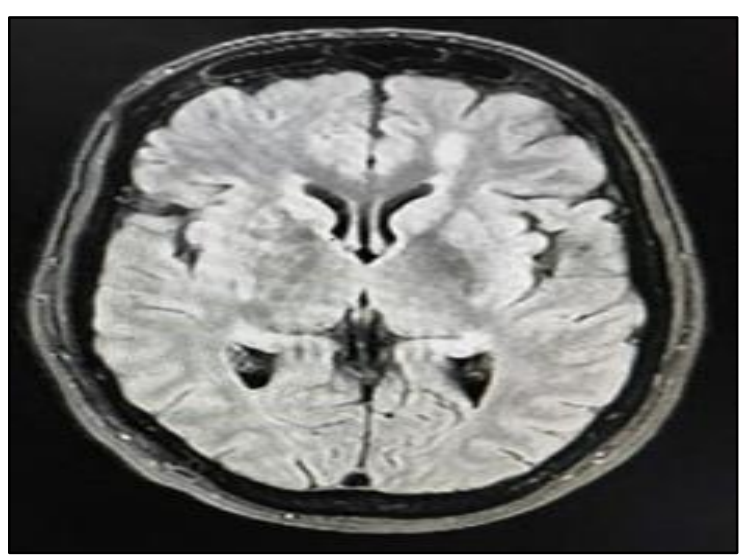

Figure 4: MRI brain- axial sections showing hyperintensity and hypo-intensity of the right caudate and right- putamen on T2-W/FLAIR images.

There is no past history of rheumatic fever, thyrotoxicosis, systemic lupus erythematosus, systemic vasculitis, autoimmune diseases, hypoxic encephalopathy, jaundice, ascites or any symptoms suggestive of portal hypertension or chronic liver disease. Serum ceruloplasmin and urinary copper levels were estimated and was found within normal range, ruling out the possibility of hepatolenticular degeneration of Wilson's disease. There was no signs and symptoms suggestive of thyrotoxicosis or parkinsonism. Her thyroid function tests were within normal limit and this rule out the possibility of thyrotoxicosis as a possible cause of Hemichorea- hemiballismus. Antinuclear antibody profile was evaluated and it came negative for certain autoimmune aetiologies including Systemic lupus erythematosus (SLE). Cerebrospinal fluid analysis was done to rule out possible intracranial infections, and all parameters of CSF analysis were within normal limit. There is no past history of oral ulcers, photosensitivity, rashes over body, joint pain, weight-loss, loss of hair or generalized weakness of the body. There was no history of recent drug intake including anti-psychotics or any addictions. None of the family members had a history of similar illness.

On admission higher mental functions were normal (MMSE-30/30) with no signs of meningeal irritation and cranial nerves were intact. No cognitive impairment was noticed. Sensory system was intact. Motor system examination revealed that she had brief, irregular, hyperkinetic, asymmetric, involuntary movements of left side of the body predominantly involving the left upper limb which progressed to involve left-lower limb and left side of face with occasional tongue movements. No signs of dysarthria, ataxia, myoclonus or tremors were noted. These abnormal movements were aggravated during activity and tends to disappear during sleep. Tone was increased on the left side showing lead type-rigidity and Power was reduced on the left side (3/5). Reflexes were 
normal. Cerebellar signs were absent and gait could not be accessed due to excessive movements. There was no bowel and bladder involvement noted.

Laboratory investigations at the time of admission revealed random plasma glucose levels of $586 \mathrm{mg} / \mathrm{dl}$ and ketone bodies were not detected in urine. Serial ABG revealed Metabolic acidosis with compensatory metabolic response. Radio-diagnostic imaging revealed hyperdensities in the head and body of right caudate and Putamen on NCCT brain. After three days of hospital stay when patient got stabilized, MRI Brain was done and it revealed hyper-intensities on T1W images and heterogenous hypo intensities in right striatum and globus pallidus T2W or Flair images, suggesting the diagnosis of non-ketotic hyperglycaemic hemichorea due to diabetic striatopathy. The patient was managed with insulin infusion therapy according to bodyweight at the rate of 5 units/hour, haloperidol $2 \mathrm{mg} /$ day, valproate $1000 \mathrm{mg} /$ day and tetrabenazine $50 \mathrm{mg} /$ day. During hospital course, adequate glycaemic control was achieved with insulin therapy and it resulted in decreasing the intensity of movement disorder but without complete resolution. After two weeks of hospital stay, she was discharged on insulin therapy besides other supportive medications like sodium valproate and tetrabanazine. In follow up, she reported to our OPD after 6 weeks of discharge and there was adequate Glycemic control and almost complete resolution of involuntary movements. The patient has signed an informed consent to allow her data publication.

\section{DISCUSSION}

Hemichorea associated with diabetes is characterized by triad of unilateral involuntary choreiform movements, contralateral striatopathy and rapid resolution of symptoms after correction of underlying hyperglycaemia. ${ }^{5}$ HCHB may be a complication in known patients with diabetes or it may be the first manifestation of the diabetes mellitus. ${ }^{6,7}$ The diagnosis is made in the context of typical clinical and radiological features including the presence of ballistic or choreiform movements in the setting of marked hyperglycemia and the absence of keto-acidosis. ${ }^{8}$ This patient had recent history of diabetes and choreiform movements decreased in intensity after strict glycaemic control after IV insulin infusion therapy besides other supportive medications like haloperidol, sodium valproate and tetrabenazine.

Neuro-imaging studies reveal characteristic features such as high densities in contralateral basal ganglia, but at times CT brain can be normal. ${ }^{9}$ MRI shows typical features such as hyper-intensities at contralateral putamen of basal ganglia in T1-weighted images and restricted diffusion in DWI. ${ }^{9}$ The exact underlying mechanism for non-ketotic hyperglycaemic chorea is unclear but various hypothesis have been proposed. ${ }^{10,11}$ The major causes include cerebral vascular insufficiency, petechial haemorrhages and depletion of neurotransmitters like acetylcholine and GABA secondary to metabolic disturbances and hyper viscosity of blood causing blood brain barrier dysfunction. ${ }^{9}$ A major hypothesis is that hyperglycaemia may directly induce alterations in dopaminergic activity and upregulation of dopamine. It was thought that hyperglycemia induces perfusion changes in the contralateral striatum as hyperglycemia shifts cerebral metabolism to an anaerobic pathway and that GABAergic neurons are particularly vulnerable to ischemia. ${ }^{1}$ The selective loss of striatal GABAergic neurons may be related to the disinhibition of thalamocortical pathway, resulting in motor cortical hyperexcitability. ${ }^{12}$ Acute putaminal dysfunction secondary to hyperosmolar state caused by hyperglycaemia and associated degeneration of putamen are considered to play a role in the pathogenesis of non-ketotic hyperglycaemic chorea. ${ }^{13}$

Recognition of this unique clinic-radiological manifestations is important because the correction of underlying hyperglycemia will lead to rapid improvement. Screening all patients who present with involuntary movements for hyperglycemia, even those without a prior history of diabetes is important. ${ }^{1}$

Prognosis of this condition was good in our patient also as the intensity of symptoms reduced as it is evident from the available literature. But it is essential to achieve glycaemic control rapidly and if failed to do so, might lead to irreversible abnormal intensities radiologically and nonresolution of abnormal movements permanently. ${ }^{8}$

\section{CONCLUSION}

Abnormal involuntary movements of acute onset with choreiform nature and characteristic MRI and CT lesions in caudate and putamen in patients with non-ketotic hyperglycaemia with or without prior history of diabetes mellitus should prompt the clinician to consider diabetic striatopathy as a cause, which might sometimes be the initial presenting symptom of underlying diabetes mellitus particularly in elderly females. Early recognition and prompt correction of underlying hyperglycaemia is essential as it will lead to rapid improvement of chorea and the prognosis is usually excellent.

\section{Funding: No funding sources \\ Conflict of interest: None declared \\ Ethical approval: Not required}

\section{REFERENCES}

1. Oh SH, Lee KY, Im JH, Lee MS. Chorea associated with non-ketotic hyperglycemia and hyperintensity basal ganglia lesion on T1-weighted brain MRI study: a meta-analysis of 53 cases including four present cases. J Neurol Sci. 2002;200(1):57-62.

2. Roy U, Das SK, Mukherjee A, Biswas D, Pan K, Biswas A, et al. Irreversible HemichoreaHemiballism in a Case of Nonketotic Hyperglycemia Presenting as the Initial Manifestation of Diabetes Mellitus. Tremor Hyperkinetic Mov. 2016;6. 
Available

at:

https://www.ncbi.nlm.nih.gov/pmc/articles/PMC5019 112/. Accessed on 23 May 2021.

3. Ohara S, Nakagawa S, Tabata K, Hashimoto T. Hemiballism with hyperglycemia and striatal T1-MRI hyperintensity: An autopsy report. Mov Disord. 2001;16(3):521-5.

4. Chorea-ballismus with nonketotic hyperglycemia in primary diabetes mellitus. American Journal of Neuroradiology. 2021. Available at: http://www.ajnr.org/content/17/6/1057.short. Accessed on 23 May 2021.

5. Qi X, Yan Y, Gao Y, Zheng Z, Chang Y. Hemichorea associated with non-ketotic hyperglycaemia: A case report. Diabetes Res Clin Pract. 2012;95(1):e1-3.

6. Crausman RS, Wen J, Al-Shalabi S. Choreoathetosis and Diabetes. Diabetes Care. 1997;20(7):1209-10.

7. Higa M, Kaneko $Y$, Inokuchi $T$. Two cases of hyperglycemic chorea in diabetic patients. Diabet Med. 2004;21(2):196-8.

8. Ray S, Howlader S, Chakraborty S, Chakraborty PP, Ghosh S. Hemichorea-Hemiballism as the First Presentation of Type 2 Diabetes. Clin Diabetes. 2015;33(2):87-9.

9. Lk S, Ak M, G M, Kp S, Sk S. Hemichorea Secondary to Non-Ketotic Hyperglycemia as the Presenting Manifestation of Diabetes Mellitus. J Assoc Physicians India. 2020;68(2):82-3.
10. Lee SH, Shin JA, Kim JH, Son JW, Lee KW, Ko SH et al. Chorea-ballism associated with nonketotic hyperglycaemia or diabetic ketoacidosis: Characteristics of 25 patients in Korea. Diabetes Res Clin Pract. 2011;93(2):e80-3.

11. Felicio AC, Chang CV, Godeiro-Junior C, Okoshi MP, Ferraz HB. Hemichorea-hemiballism as the first presentation of type 2 diabetes mellitus. Arq Neuropsiquiatr. 2008;66(2A):249-50.

12. Hemichorea-hemiballism: an explanation for MR signal changes. American Journal of Neuroradiology. Available at: http://www.ajnr.org/content/19/5/863.short. Accessed on 23 May 2021.

13. Unilateral Putaminal CT, MR, and Diffusion Abnormalities Secondary to Nonketotic Hyperglycemia in the Setting of Acute Neurologic Symptoms Mimicking Stroke. American Journal of Neuroradiology. Available at: http://www.ajnr.org/content/25/6/975.short. Accessed on 23 May 2021.

Cite this article as: Kumar V, Patel KVK, Raj R, Bhushan D, Hegde AV. Hemichorea-hemiballismus as a rare presentation of non-ketotic hyperglycemia: a case report. Int J Basic Clin Pharmacol 2022;11:181-4. 